\title{
Serious game training in medical education: potential to mitigate cognitive biases of healthcare professionals
}

https://doi.org/10.1515/dx-2021-0004

Published online February 22, 2021

Keywords: clinical reasoning; cognitive bias; debiasing; health professions education; serious games.

"Whenever there is a simple error that most laymen fall for, there is always a slightly more sophisticated version of the same problem that experts fall for."

Amos Tversky

Decision making is at the heart of medical practice [1-3]; the clinical reasoning process has been researched extensively over the past three decades [1-7]. Decision making in healthcare can be influenced by different factors affecting the diagnostic problem space, including the environment, the situation, and the culture and norms of the community of practice. For example, stress negatively influences clinicians' diagnostic accuracy $[2,3,7]$, and predisposes to premature closure $[5,6,8,9]$.

Cognitive biases have been extensively researched in recent decades [1-7]. Haselton et al. define cognitive bias as a deviation from rational, normative judgment [10]. Cognitive biases are encountered in every field and discipline, including economics, the behavioural sciences, law, medicine, forensics, and the social sciences $[2,5,6,11,12]$. Many

\footnotetext{
*Corresponding author: Julia Sader, PhD Candidate, UDREM - Unit of Development and Research in Medical Education, Faculty of Medicine, University of Geneva, Geneva, Switzerland; and iEH2 - Institute for Ethics, History, and the Humanities, Faculty of Medicine, University of Geneva, Geneva, Switzerland, E-mail: Julia.sader@unige.ch

Christine Clavien and Samia Hurst, iEH2 - Institute for Ethics, History, and the Humanities, Faculty of Medicine, University of Geneva, Geneva, Switzerland

James Korris, CTI - Creative Technologies, Inc., Los Angeles, CA, USA Mathieu Nendaz, UDREM - Unit of Development and Research in Medical Education, Faculty of Medicine, University of Geneva, Geneva, Switzerland; HUG - Department of Medicine University Hospitals Geneva, Geneva, Switzerland

Marie-Claude Audétat, UDREM - Unit of Development and Research in Medical Education, Faculty of Medicine, University of Geneva, Geneva, Switzerland
}

Әopen Access. ( 2021 Julia Sader et al., published by De Gruyter. ((c) BY License. studies $[3-7,11]$ have explored how to mitigate these biases while expanding our understanding of their processes as well as their impact on our decision making. Daniel Kahneman and Amos Tversky argue that cognitive biases have significant practical implications for areas including clinical judgment, finance and management [6]. The hypothesis that one can "get rid of" one's biases reflects a simplistic perspective regarding these ingrained, automatic, and subconscious tendencies. Indeed, many cognitive scientists regard these biases as generally adaptive, even though they can also predispose to diagnostic errors [6, 7]. Mamede and others have explored the use of clinical vignettes to promote reflection as one approach to minimize the impact of certain biases on diagnostic reasoning $[2,4,5]$.

The COVID-19 pandemic has emphasized how external factors can adversely affect clinical decision-making [13]. The sparsity of resources, the constant threat of infection, and the psychological stress of caring for so many dying patients are all factors that could detract from optimal decision-making. The pandemic may also predispose to cognitive bias [14]. Given the overwhelming number of patients with COVID-19 infection, one might easily assume that a patient presenting with fever has the disease and miss a timely diagnosis of sepsis from some other cause (availability bias).

Serious games are games which, besides entertainment, have another purpose, generally educational. They are designed to solve problems and include challenges and rewards, using the components of entertainment and interaction to provide education while playing a game $[8,9,11$, 12, 15]. Different industries and fields have adapted serious games for their own purposes, from teaching new employees how to use a software to teaching teenagers the risks of drinking and driving [8-11, 13, 14]. Barton \& Symborski et al. have described the effectiveness of such serious game training interventions in the field of behavioural sciences [11]. The nature of these individual differences, combined with the ability to judge and make choices through training techniques, provides promising proof that it is possible to develop an awareness of and mitigation tools for cognitive 
biases over the long term. Indeed, the effect of cognitive biases such as vulnerability to anchoring, confirmation bias and bias blind spot has been reduced through debiasing serious game training interventions $[11,16]$.

Theoretical foundations in the development of serious games in healthcare are crucial. To expand on this, the serious game to mitigate cognitive biases [11] has two key steps, firstly decipher the learning stages and secondly describe the cognitive learning opportunities arising from the levels of proficiency [11]. This implies that when designing a game, the pedagogical strategies intended by the authors of the game has a direct impact on the architecture of the games $[8,9,12,15]$.

Serious gaming has the potential to be a useful pedagogical tool which could benefit medical education. Gentry et al. and Gorbanev et al. provide an objective assessment of serious games and found that when the objectives of such games are clear, they have a positive outcome in knowledge acquisition [9, 15]. They also point out the importance of embedding these tools in a coherent and reflected pedagogical framework - lack of prior reflection may result in a loss of efficacy [15]. The literature shows a promising landscape for the effectiveness of serious games in medical education. However, despite these promising prospects, serious games are not always seen as the most relevant method for medical teaching. A variety of reasons could explain this, such as the fact that serious games need to build on clear branching scenarios with simple inputoutput structures. This may be perceived as a tooconstrained setup for unclear learning objectives [9, 15]. Beyond the game itself, both reviews convey the possible ethical issues which may arise, resulting from uneven resources availability which impedes the prospect for significant implementation.

Developing the skills to recognize and mitigate cognitive biases diverges from the kinds of curricula generally found in games for medical training which typically focus on declarative medical knowledge and part task/procedural medical instruction [9, 12, 15]. As these are thinking skills - not narrowly medical skills - a game about cognitive biases would require agile transferability, such as seen in Sellier et al. [16]. This should also be addressed in future studies.

Given the present knowledge regarding serious games in medical education, thorough quantitative research should be undertaken in order to showcase how training interventions through the use of serious games can influence cognitive biases in the medical field and amongst healthcare professionals.

Research funding: None declared.
Author contributions: All authors have accepted responsibility for the entire content of this manuscript and approved its submission.

Competing interests: Authors state no conflict of interest. Ethical approval: Not applicable.

\section{References}

1. Audétat MC, Laurin S, Dory V, Charlin B, Nendaz MR. Diagnosis and management of clinical reasoning difficulties: part I. Clinical reasoning supervision and educational diagnosis. Med Teach 2017;39:792-6.

2. Mamede S, van Gog T, van den Berge K, Rikers R, van Saase J, van Guldener C, et al. Effect of availability bias and reflective reasoning on diagnostic accuracy among internal medicine residents. JAMA 2010;304:1198-203.

3. Nendaz M, Perrier A. Diagnostic errors and flaws in clinical reasoning: mechanisms and prevention in practice. SMW 2012:142.

4. Croskerry P, Singhal G, Mamede S. Cognitive debiasing 1: origins of bias and theory of debiasing. BMJ Qual Saf 2013;22(2 Suppl): ii58-64.

5. Norman GR, Monteiro SD, Sherbino J, Ilgen JS, Schmidt HG, Mamede $S$. The causes of errors in clinical reasoning: cognitive biases, knowledge deficits, and dual process thinking. Acad Med 2017;92:23-30.

6. Tversky A, Kahneman D. The framing of decisions and the psychology of choice. Science 1981;211:453-8.

7. Wright $B$, Faulkner N, Bragge $P$, Graber $M$. What interventions could reduce diagnostic error in emergency departments? A review of evidence, practice and consumer perspectives. Diagnosis 2019;6:325-34.

8. Dunbar NE, Jensen ML, Miller CH, Bessarabova E, Straub SK, Wilson SN, et al. Mitigating cognitive bias through the use of serious games: effects of feedback. In Persuasive Technology 9th International Conference, PERSUASIVE 2014, Proceedings (pp. 92-105).

9. Gentry SV, Gauthier A, Ehrstrom BL, Wortley D, Lilienthal A, Car LT, et al. Serious gaming and gamification education in health professions: systematic review. J Med Internet Res 2019;21:e12994.

10. Haselton MG, Nettle D, Andrews PW. The evolution of cognitive bias. In: Buss DM, editor. The handbook of evolutionary psychology. New Jersey: John Wiley \& Sons; 2005.

11. Barton M, Symborski C, Quinn M, Morewedge CK, Kassam KS, Korris $\mathrm{JH}$. The use of theory in designing a serious game for the reduction of cognitive biases. Trans Digit Game Res Assoc 2016;2.

12. Haoran G, Bazakidi E, Zary N. Serious games in health professions education: review of trends and learning efficacy. Yearb Med Inf 2019;28:240.

13. Audétat MC, Sader J, Coen M. Clinical reasoning and COVID 19 pandemic: current influencing factors Let us take a step back! Intern Emer Med 2020:1-3. https://doi.org/10.1007/s11739-020-02516-8.

14. Brown L. Covid blindness. Dignosis 2020;7:83-4.

15. Gorbanev I, Agudelo-Londoño S, González RA, Cortes A, Pomares A, Delgadillo V, et al. A systematic review of serious games in medical education: quality of evidence and pedagogical strategy. Med Educ Online 2018;23:1438718.

16. Sellier AL, Scopelliti I, Morewedge CK. Debiasing training improves decision making in the field. Psyc Sci 2019;30:1371-9. 